\section{Quantum tunnelling towards an exploding Universe?}

\author{
from Malcolm J. Perry
}

THE discovery that superstring theory might enable one to form a consistent quantum theory of gravitation unified with the other fundamental forces - the strong, weak and electromagnetic interactions of matter - has generated an excitement in the theoretical physics community unparalleled since the invention of quantum electrodynamics (see Green, M.B. Nature 314, 409; 1985 for a review of recent superstring theories). Nevertheless, string theory does not yet yield a complete picture of the physical world as we know it. One problem is that it predicts that the Universe has either 10 or 26 space-time dimensions, in contrast with the 4 space-time dimensions in which we seem to live. What is the fate of the remaining 6 or 22 dimensions?

The idea that space-time has more than four dimensions was originally explored by Th. Kaluza and $\mathrm{O}$. Klein during the 1920s. These authors produced a unified classical theory of gravitation and electromagnetism in which space-time was fivedimensional, being the product of a circle of very small spatial extent and an ordinary four-dimensional space-time. In this theory, the fundamental unit of electrical charge $e$ is related to the circumference $l$ of the Kaluza-Klein circle by

$$
l=\frac{1}{e} \sqrt{64 \pi^{3} G}
$$

where $G$ is Newton's constant. Thus, in this theory, $l=2.3 \times 10^{-31} \mathrm{~cm}$. The theory has the advantage of predicting that electric charge is quantized. To probe such short distance scales, and hence to observe them directly, would require incredibly large energies of about $10^{19} \mathrm{GeV}$. Thus, we need never worry about the fifth dimension in ordinary experiments. But this simple model turned out to be unrealistic because it predicts that charged particles must be very massive, again $\sim 10^{15}$ $\mathrm{GeV}$. But a beautiful feature of the theory was that it was based purely on fivedimensional general relativity, only adding the assumption that space-time was partially wrapped up.

Today, a Kaluza-Klein theory refers to any model in which space-time is partially compactified, making some dimensions of small spatial extent. These small 'compact' dimensions will always be hard to observe directly, but they have profound indirect consequences. This compactification is presumably required in string theory. In the original Kaluza-Klein example the circle gave rise to electromagnetism. In more complicated examples based on $M^{(t)} \times \Sigma^{(d)}$, where $M^{(4)}$ is the large four-dimensional space-time and $\Sigma^{(t)}$ is a $d$-dimensional compact space, the isometry group of $\Sigma$ manifests itself as the low-energy gauge group of a Yang-Mills theory (a relativistic point-field theory). Thus, in the original example, the iso- metry group of the circle $\mathrm{S}^{1}$ is $\mathrm{U}(1)$. We can generate many gauge groups in this way, for example $\mathrm{SO}(n)$ emerges if $\Sigma$ is $\mathrm{S}^{n-1}$, and $\mathrm{SU}(n)$ emerges if $\Sigma$ is $\mathrm{CP}^{n-1}$. In all cases we get a Yang-Mills-type theory with a coupling constant $g$ inversely proportional to the linear dimensions of the compact space. The difficulty with such theories is that there is usually no natural mechanism to make space-time look like $\mathbf{M}^{(4)} \times \Sigma^{(d)}$, as opposed to $\mathbf{M}^{(6)} \times \Sigma^{(d-2)}$ or $\mathbf{M}^{(4+d)}$. If there are solutions that look fourdimensional on large scales, one can argue that such a space-time is appropriate and perfectly satisfactory. Such an anthropic argument is unconvincing because there are other equally plausible solutions that do not correspond to the Universe as we observe it.

So far, I have based the discussion on classical mechanics. Recently, E. Kolb and J. Frieman (Phys. Rev. Lett. 5, 1435; 1985) have shown that when quantum mechanics it taken into account, such an argument loses all its appeal. They observe that although a space-time of the form $M^{(4)} \times \Sigma^{(d)}$ may well exist, a solution of the field equations looking like $M^{(4+d)}$ will also usually exist. $M^{(4+d)}$ is typically a $(4+d)$-dimensional version of de Sitter space, a maximally symmetrical spacetime, whose spatial extent is exponentially expanding on a timescale $\tau$. Classically, a transition from one type of solution to the other is forbidden by the existence of a large potential barrier. In quantummechanical terms, parts of the Universe can tunnel from the desired state to a $(4+d)$-dimensional de Sitter space. The probability of a transition per unit volume per unit time is calculated to be

$$
\Gamma \sim \frac{1}{l_{p}^{4}} \exp \left(-c \tau^{2} / l_{p}^{2}\right)
$$

where $l_{p}$ is the Planck length (typical of gravitational phenomena) $1.6 \times 10^{-33} \mathrm{~cm}$ and $c$ is a constant. Both are determined by the detailed dynamics of the theory, but typically $1 \leqslant c \leqslant 100$ and $\tau$ is around 1 to 10 Planck times. (One Planck time is the time taken for light to travel one Planck length, and is $5.3 \times 10^{-44} \mathrm{~s}$.)

Thus, if $c$ and $\tau$ have appropriate values, we can live in state which is $M^{(4)} \times$ $\Sigma^{(d)}$ and can be reasonably certain that we will remain there for several times the observed age of the Universe. But it is possible that $c$ and $\tau$ are such that $\Gamma$ becomes so large that it is likely to tunnel into a state in which the Universe is $M^{(4+d)}$. Here, space would be $3+d$-dimensional, expanding exponentially on the very short timescale $\tau$. This unfortunate catastrophe would, like all quantum phenomena, occur completely at random. A calculation of $c$ and $\tau$ in string theory then presents an interesting challenge. 